\title{
Identifiability and Estimation of Probabilities from Multiple Databases with Incomplete Data and Sampling Selection
}

\author{
Jinzhu Jia, Zhi Geng, and Mingfeng Wang \\ School of Mathematical Sciences, LMAM, Peking University, Beijing 100871, China
}

\begin{abstract}
For an application problem, there may be multiple databases, and each database may not contain complete variables or attributes, that is, some variables are observed but some others are missing. Further, data of a database may be collected conditionally on some designed variables. In this paper, we discuss problems related to data mining from such multiple databases. We propose an approach for detecting identifiability of a joint distribution from multiple databases. For an identifiable joint distribution, we further present the expectation-maximization (EM) algorithm for calculating the maximum likelihood estimates (MLEs) of the joint distribution.
\end{abstract}

\section{Introduction}

With development and popularity of computers, various databases have been built, which contain different variables or attributes, and whose data are collected in different conditions. For example, in medical research, some researchers collect data of these variables, but other researchers may collect data of other variables; On the other hand, some data are from follow-up studies, but other data may be from case-control studies. Distributions of diseases and associations among variables should be evaluated by combining all databases from different researches. There are several statistical approaches for combining multiple databases, such as file-matching for large databases and split questionnaire survey sampling [5, 6. Multiple databases are depicted as a hypergraph in 1, 4]. In these approaches, a database involving a subset of variables is treated as a sample from a marginal distribution of these variables. In this paper, we consider that a database involving a subset of variables may be a sample drawn conditionally on some other variables, called designed variables.

Identifiability and estimation of a joint distribution of variables are two important problems for data mining from multiple databases. In this paper, we show conditions for identifiability of a joint distribution from marginal and conditional distributions of observed variables, and we propose an approach for detecting identifiability. For an identifiable joint distribution, we present the expectationmaximization (EM) algorithm for calculating the maximum likelihood estimates (MLEs) of the joint distribution [3, 4. In the partial imputation EM algorithm [4], there must be a database containing all variables and other databases are 
drawn from marginal distributions. In this paper, we show that the database containing all variables may be unnecessary for identifying the joint distribution of all variables, and our algorithm for finding MLEs also deals with databases drawn from conditional distributions.

Section 2 gives notation and definitions. In Section 3, we show conditions for identifiability of a joint distribution from multiple databases. In Section 4, we apply the EM algorithm to calculate MLEs of the joint distribution. Section 5 gives a numerical example to illustrate our approach. Finally, Section 6 presents a simulation to evaluate the estimates.

\section{Notation and Definitions}

Let $V=\left\{x_{1}, \ldots, x_{p}\right\}$ denote the set of all variables included in an interested system, and assume that all variables in $V$ are discrete. Let $p(V=v)$ denote the distribution of variables in $V$. Suppose that there are $K$ databases, $D_{1}, \ldots, D_{K}$. For the database $D_{k}$, let $A_{k}$ denote the set of designed variables which is used to stratify the population into subpopulations with different values of $A_{k}$, let $B_{k}$ denote the set of observed variables, and let $V_{k}=A_{k} \cup B_{k}$ represent the set of variables involved in the database $D_{k}$. Denote the database $D_{k}$ as $\left[B_{k} \mid A_{k}\right]$, which means that variables in $B_{k}$ are observed conditionally on variables in $A_{k}$. For the database $D_{k}$, let $n_{k}\left(b_{k} \mid a_{k}\right)$ denote the frequency of observed individuals with value $B_{k}=b_{k}$ in the subpopulation of $A_{k}=a_{k}$. Below we first give a general definition of identifiability [2].

Definition 1. Consider a vector $Y$ of random variables having a distribution $F(y ; \theta)$ that depends on an unknown parameter vector $\theta$. $\theta$ is identifiable by observation of $Y$ if distinct values for $\theta$ yield distinct distributions of $Y$, that is, $\theta_{1} \neq \theta_{2} \Rightarrow F\left(y ; \theta_{1}\right) \neq F\left(y ; \theta_{2}\right)$.

Informally, identifiability means that these databases contain sufficient information such that the joint distribution can be uniquely determined. In the case of multiple databases, we say that the joint distribution $P(V)$ is identifiable by databases $\left[B_{1} \mid A_{1}\right] \ldots,\left[B_{K} \mid A_{K}\right]$ if distinct values for $P(V)$ yield distinct values for vector $\left(P\left(B_{1} \mid A_{1}\right), P\left(B_{2} \mid A_{1}\right), \cdots, P\left(B_{K} \mid A_{K}\right)\right)$.

\section{Conditions for Identifiability from Multiple Databases}

In this section, we show a condition for identifiability of the joint distribution of $V$ from multiple databases $\left[B_{1} \mid A_{1}\right], \ldots,\left[B_{K} \mid A_{K}\right]$. We first show several lemmas which are used to prove the condition for identifiability.

Lemma 1. Suppose that there are only two databases $D_{1}=[B \mid A]$ and $D_{2}=$ $[A \mid B]$ where $A \cup B=V$. Then the joint distribution $P(V)$ of variables in $V$ is identifiable.

Proof. Since $P(B \mid A) / P(A \mid B)=P(B) / P(A)$, we have $\sum_{B}[P(B \mid A) / P(A \mid B)]=$ $\sum_{B}[P(B) / P(A)]=1 / P(A)$. Thus we can find $P(A, B)=P(B \mid A) P(A)=$ 
$P(B \mid A) /\left[\sum_{B} P(B \mid A) / P(A \mid B)\right]$. Since $P(A, B)$ is a function of $P(A \mid B)$ and $P(B \mid A)$ and since they are identifiable by the databases $[A \mid B]$ and $[B \mid A]$ respectively. So we get that $P(A, B)$ is identifiable by the databases $[B \mid A]$ and $[A \mid B]$.

Lemma 2. Suppose that there are only two databases $D_{1}=\left[B_{1} \mid A_{1}\right]$ and $D_{2}=$ $\left[B_{2} \mid A_{2}\right]$ and that $A_{i} \cup B_{i}=V$ for $i=1$ and 2 . Define $A=A_{1} \cap A_{2}$ and $B=V \backslash A$. Then the conditional probability $P(B \mid A)$ is identifiable by the databases, where the conditional probability $P(B \mid \emptyset)$ is defined as the marginal probability $P(B)$.

Proof. If $A=\emptyset$, then the result can be obtained immediately from Lemma 1 . Below we consider the case of $A \neq \emptyset$. Define $C_{1}=A_{1} \backslash A$ and $C_{2}=A_{2} \backslash A$. Then $A_{1}=C_{1} \cup A, A_{2}=C_{2} \cup A, C_{1} \cap C_{2}=\emptyset$ and $C_{i} \cap A=\emptyset$ for $i=1,2$. We have

$$
\frac{P\left(B_{1} \mid A_{1}\right)}{P\left(B_{2} \mid A_{2}\right)}=\frac{P\left(A_{2}\right)}{P\left(A_{1}\right)}=\frac{P\left(C_{2}, A\right)}{P\left(C_{1}, A\right)} .
$$

Thus,

$$
\sum_{C_{2}} \frac{P\left(B_{1} \mid A_{1}\right)}{P\left(B_{2} \mid A_{2}\right)}=\sum_{C_{2}} \frac{P\left(C_{2}, A\right)}{P\left(C_{1}, A\right)}=\frac{P(A)}{P\left(C_{1}, A\right)}=\frac{1}{P\left(C_{1} \mid A\right)} .
$$

Further, we have

$$
P\left(B_{1} \mid A_{1}\right) P\left(C_{1} \mid A\right)=P\left(B_{1} \mid C_{1}, A\right) P\left(C_{1} \mid A\right)=P\left(B_{1}, C_{1} \mid A\right)=P(B \mid A) .
$$

We get that $P(B \mid A)$ is a function of $P\left(B_{1} \mid A_{1}\right)$ and $P\left(A_{1} \mid B_{1}\right)$ and thus $P(B \mid A)$ is identifiable by $\left[B_{1} \mid A_{1}\right]$ and $\left[B_{2} \mid A_{2}\right]$.

Lemma 3. Suppose that there are $K$ databases, $\left[B_{1} \mid A_{1}\right], \ldots,\left[B_{K} \mid A_{K}\right]$, and $A_{k} \cup$ $B_{k}=V$ for all $k$. The joint distribution $P(V)$ is identifiable if $\bigcap_{i=1}^{n} A_{i}=\emptyset$.

Proof. This result can be obtained immediately by applying Lemma 2 repeatedly to each pair of databases.

Now we propose an algorithm for detecting identifiability of the joint distribution $P(V)$ from $K$ databases, $\left[B_{1} \mid A_{1}\right], \ldots,\left[B_{K} \mid A_{K}\right]$, where $A_{k} \cup B_{k} \subseteq V$.

Algorithm: Detect identifiability of $P(V)$ from the $K$ databases.

1. Initialize $t=0,\left[B_{k}^{(0)} \mid A_{k}^{(0)}\right]=\left[B_{k} \mid A_{k}\right]$ for all $k$, and $V^{(0)}=V$. Below we repeatedly check whether $P\left(V^{(t)}\right)$ is identifiable by databases $\left[B_{k}^{(t)} \mid A_{k}^{(t)}\right]$ for all $k$.

2. Find a database $\left[B_{i}^{(t)} \mid A_{i}^{(t)}\right]$ such that $B_{i}^{(t)} \cup A_{i}^{(t)}=V^{(t)}$. If there is no such a database, then $P(V)$ is not identifiable and stop the algorithm.

3. Let $V^{(t+1)}=A_{i}^{(t)},\left[B_{k}^{(t+1)} \mid A_{k}^{(t+1)}\right]=\left[B_{k}^{(t)} \backslash B_{i}^{(t)} \mid A_{k}^{(t)} \backslash B_{i}^{(t)}\right]$ for all $k$, and $t=t+1$.

4. Repeat Steps 2 and 3 until $V^{(t)}=\emptyset$. 
If the algorithm returns an empty set $V^{(t)}$, then the probability $P(V)$ is identifiable. Below we first give an example to illustrate the algorithm, and then we show the correctness of the algorithm.

Example 1. Consider that there are seven variables $V=\{1,2,3,4,5,6,7\}$ and five databases [456|1237], [147|23], [45|6], [2|1347], [3|45]. We apply the above algorithm to the databases to detect identifiability of $P(V)$.

For the first iteration, at Step 2, we find $\left[B_{1} \mid A_{1}\right]=[456 \mid 1237]$ with $A_{1} \cup B_{1}=$ $V$. At Step 3, we reset the set to be identified as $V^{(1)}=A_{1}=[1237]$, remove variables 4,5 and 6 in $B_{1}$ from all databases and obtain databases for the next iteration as [17|23], [2|137] and [3]. For the second iteration, at Step 2, we find $A_{1}^{(1)} \cup B_{1}^{(1)}=V^{(1)}=[1237]$. At Step 3, set $V^{(2)}=A_{1}^{(1)}=[23]$, remove variables 1 and 7 in $B_{1}^{(1)}$ and obtain databases [2|3], [3]. For the third iteration, we get $V^{(3)}=[3]$ and database [3]. For the fourth iteration, we get $V^{(4)}=\emptyset$ and thus we say that $\mathrm{P}(\mathrm{V})$ is identifiable.

Lemma 4. Suppose that there are two databases $\left[B_{1} \mid A_{1}\right]$ and $\left[B_{2} \mid A_{2}\right]$. Define $V_{i}=A_{i} \cup B_{i}$ for $i=1$ and $2, V_{0}=V_{1} \cap V_{2}$ and $A=A_{1} \cap A_{2}$. Then $P\left(V_{0} \backslash A \mid A\right)$ is identifiable by the two databases if $V_{1} \backslash V_{2} \subseteq B_{1}$ and $V_{2} \backslash V_{1} \subseteq B_{2}$.

Proof. From $\left[B_{1} \mid A_{1}\right]$ we can get $P\left(B_{1} \mid A_{1}\right)$, and in turn we can obtain $P\left(B_{1} \backslash\right.$ $\left.\left(V_{1} \backslash V 2\right) \mid A_{1}\right)$. Similarly we can obtain $P\left(B_{2} \backslash\left(V_{2} \backslash V 1\right) \mid A_{2}\right)$. From the conditions $V_{1} \backslash V 2 \subseteq B_{1}$ and $V_{2} \backslash V_{1} \subseteq B_{2}$, we have $A_{1} \subseteq V_{2}$ and $A_{2} \subseteq V_{1}$ respectively. Thus we get $B_{1} \backslash\left(V_{1} \backslash V_{2}\right) \cup A_{1}=V_{1} \cap V_{2}$ and $B_{2} \backslash\left(V_{2} \backslash V 1\right) \cup A_{2}=V_{1} \cap V_{2}$. By Lemma 2, we know that $P\left(V_{0} \backslash A \mid A\right)$ can be obtained.

Corollary 1. Suppose that $V_{2} \subseteq V_{1}$ and $A_{1}$ can be partitioned into two sets $A$ and $B$ (that is, $A_{1}=A \cup B$ and $A \cap B=\emptyset$ ) such that $A \subseteq A_{2}$ and $B \subseteq B_{2}$. Then $P(B \mid A)$ is identifiable and thus $P\left(V_{1} \backslash A \mid A\right)$ is identified.

Proof. Since $A_{1}=A \cup B$, we get $V_{2}=A_{2} \cup B_{2} \supseteq A \cup B=A_{1}$. Thus we have $V_{1} \backslash V_{2} \subseteq B_{1}$. From supposition we have $V_{2} \backslash V_{1}=\emptyset \subseteq B_{2}$. From Lemma 4, we obtain that $P(B \mid A)$ is identifiable. We have

$$
P\left(B_{1} \mid A_{1}\right) \times P(B \mid A)=\frac{P\left(V_{1}\right)}{P\left(A_{1}\right)} \times \frac{P\left(A_{1}\right)}{P(A)}=P\left(V_{1} \backslash A \mid A\right) .
$$

Thus we showed that $P\left(V_{1} \backslash A \mid A\right)$ is identifiable.

Example 2. Suppose that $V=\{1,2,3,4,5,6,7\}=V_{1}$ and $V_{2}=\{1,2,4,5,7\}$ and that we have two databases $\left[B_{1} \mid A_{1}\right]=[1236 \mid 457]$ and $\left[B_{2} \mid A_{2}\right]=[24 \mid 157]$. Partition $A_{1}$ as $A=\{5,7\}$ and $B=\{4\}$. Since $A \subseteq A_{2}$ and $B \subseteq B_{2}$, we get from Corollary 1 that $P(4 \mid 57)$ and $P(1,2,3,4,6 \mid 57)$ are identifiable.

Theorem 1. The probability $P(V)$ is identifiable by databases $\left[B_{1} \mid A_{1}\right], \ldots$, $\left[B_{K} \mid A_{K}\right]$ if the algorithm returns an empty set $V^{(t)}$. 
Proof. We first show the result following the iterations of the algorithm. At the first iteration, we have immediately that $P\left(B_{i}^{(0)} \mid A_{i}^{(0)}\right)$ is identifiable by the database $\left[B_{i}^{(0)} \mid A_{i}^{(0)}\right]$ where $A_{i}^{(0)} \cup B_{i}^{(0)}=V$. At the second iteration, we partition $V^{(1)}=A_{i}^{(0)}$ into two sets $A_{i}^{(1)}$ and $B_{i}^{(1)}$, which must be contained by $A_{k}$ and $B_{k}$ of some original database $\left[B_{k} \mid A_{k}\right]$ respectively. By Corollary 1 , we have that $P\left(V \backslash A_{i}^{(1)} \mid A_{i}^{(1)}\right)$ is identifiable. Following the iterations, we can obtain that $P\left(V \backslash A_{i}^{(t)} \mid A_{i}^{(t)}\right)$ is identifiable. If $A_{i}^{(t)}=\emptyset$ is obtained at the $t$-th iteration, then $P(V)$ is identifiable.

\section{Maximization Likelihood Estimation of Probabilities}

For a joint probability $P(V)$ which is identifiable, we propose the EM algorithm for calculating MLEs from databases $\left[B_{1} \mid A_{1}\right], \ldots,\left[B_{K} \mid A_{K}\right]$. Data in each database, say $D_{k}=\left[B_{k} \mid A_{k}\right]$, may be incomplete (i.e. $A_{k}$ is a pure subset of $V$ ), and they may be obtained conditionally on designed variables $B_{k}$. For simplicity, we show only a simple case of two variables $V=\{X, Y\}$ and two databases $[X \mid Y]$ and $[Y \mid X]$. The algorithm can be easily extended to a general case.

Let $n_{1}(x \mid y)$ denote the observed frequency of individuals with $X=x$ conditional on $Y=y$ in the first database for $x=1, \ldots, I$; and let $n_{2}(y \mid x)$ denote that with $Y=y$ conditional on $X=x$ in the second database for $y=1, \ldots, J$. Below we present the EM algorithm for calculating MLEs $\left\{\hat{p}_{x y}\right\}$ of the joint probabilities $P(X=x, Y=y)$ for all $x$ and $y$. We treat the frequencies $\left\{n_{1}(x \mid y), \forall x\right\}$ as the $y$-th column of an $I \times J$ contingency table $\left\{n_{1}^{(y)}(i, j) \forall i, j\right\}$. Similarly, $\left\{n_{2}(y \mid x), \forall y\right\}$ as the $x$-th row of an $I \times J$ contingency table $\left\{n_{2}^{(x)}(i, j), \forall i, j\right\}$. Assume that the total frequency $n_{k}^{(m)}=\sum_{i, j} n_{k}^{(m)}(i, j)$ follows a Poisson distribution with parameter $\lambda_{k}(m)$ and that $\left\{n_{k}^{(m)}(i, j), \forall i, j\right\}$ follows a multinomial distribution with parameters $\left\{p_{i j}\right\}$ given a total frequency $n_{k}^{(m)}$.

At the E-step of the EM algorithm, we find the expected frequencies of $\left\{n_{1}^{(y)}(i, j), \forall i, j\right\}$ and $\left\{n_{2}^{(x)}(i, j), \forall i, j\right\}$. At the M-step, we find the MLEs $\left\{\hat{p}_{i j}, \forall i, j\right\}$ and $\left\{\lambda_{k}(m), \forall k, m\right\}$. The EM algorithm for calculating MLEs is given below.

1. E-step:

$$
\begin{aligned}
\hat{n}_{k}^{(m)}(i, j) & =E\left[n_{k}^{(m)}(i, j) \mid\left\{n_{1}(x \mid y)\right\},\left\{n_{2}(y \mid x)\right\},\left\{\lambda_{k}^{(t)}(m)\right\},\left\{p_{i j}^{(t)}\right\}\right] \\
& =\lambda_{k}^{(t)}(m) \times p_{i j}^{(t)}
\end{aligned}
$$

for $i, j, k$ and $m$.

2. M-step:

$$
\begin{aligned}
& \hat{\lambda}_{k}^{(t+1)}(m)=\sum_{i, j} \hat{n}_{k}^{(m)}(i, j), \\
& \hat{p}_{i j}^{(t+1)}=\frac{1}{N} \sum_{k, m} \hat{n}_{k}^{(m)}(i, j),
\end{aligned}
$$


where the total frequency $N$ equals $\sum_{k, m, i, j} \hat{n}_{k}^{(m)}(i, j)$.

3. Repeat the E-step and M-step until some convergence criterion is achieved.

\section{A Numerical Example}

In this section, we use an artificial data to illustrate our approach. Suppose that we have a case-control study and a follow-up study on association between smoking and lung cancer, as shown in Tab. 1. In the case-control study with databases $[X \mid Y]$, we have 709 cases and 709 controls, and then ask their smoking history. In the follow-up study with databases $[Y \mid X]$, we have a smoker group of 2000 individuals and a non-smoker group of 2000 individuals, and follow their states for years. Combining these databases, we can identify the joint distribution of $X$ and $Y$ and the marginal distributions of $X$ and $Y$ (see Tab. 1), any of which is not identifiable by using only one of $[X \mid Y]$ and $[Y \mid X]$. Especially, the MLE of the relative risk, $\hat{P}(Y=1 \mid X=1) / \hat{P}(Y=1 \mid X=0)=3.1160$, can be found by combining all data in these databases. It is more efficient than the MLE of the relative risk $(8 / 2000) /(2 / 2000)=4$ obtained by using data only from the follow-up study since there are a few of cancer cases in the follow-up study.

Table 1. A case-control study and a follow-up study on smoking and lung cancer

\begin{tabular}{|c|c|c|c|c|c|c|c|}
\hline & \multicolumn{3}{|c|}{ Case-control study } & \multicolumn{2}{|c|}{ Follow-up study } & \multicolumn{2}{|c|}{ MLEs of probabilities } \\
\hline & Smoker & on-smok & Total & Smoker & Non-smoker & Smoker & Non-smoker \\
\hline & $X=1$ & $X=0$ & & $X=1$ & $X=0$ & $X=1$ & $X=0$ \\
\hline Cancer $(Y=1)$ & 688 & 21 & 709 & 8 & 2 & 0.0067 & 0.0002 \\
\hline Control $(Y=0)$ & 650 & 59 & 709 & 1992 & 1998 & 0.9082 & 0.0849 \\
\hline Total & & & & 2000 & 2000 & & \\
\hline
\end{tabular}

\section{Simulation}

Consider two binary variables $V=\left\{X_{1}, X_{2}\right\}$ with values 0 and 1 . Suppose that there are two databases $\left[X_{1} \mid X_{2}\right]$ and $\left[X_{2} \mid X_{1}\right]$. From the results in Section 3 , we know that the joint probabilities of $X_{1}$ and $X_{2}$ are identifiable from these databases. Below we give a numerical simulation to illustrate estimation of the joint probability $P\left(x_{1}, x_{2}\right)$. From the true distribution given in Tab. 2,

Table 2. True probabilities and their estimates

\begin{tabular}{|c|c|c|c|}
\hline & & \multicolumn{2}{|r|}{ Estimates } \\
\hline & True value & Mean & Standard error \\
\hline$\overline{p_{00}}$ & 0.1000 & 0.0993 & 0.0230 \\
\hline$p_{01}$ & 0.2000 & 0.2004 & 0.0372 \\
\hline$p_{10}$ & 0.3000 & 0.3007 & 0.0499 \\
\hline$p_{11}$ & 0.4000 & 0.3994 & 0.0507 \\
\hline
\end{tabular}


we generate each of databases $\left[X_{1}=i \mid X_{2}=j\right]$ and $\left[X_{2}=j \mid X_{1}=i\right]$ for all $i$ and $j$ from a binomial distribution with sample size 50. Then we apply the EM algorithm to calculate MLEs $\hat{p}_{i j}$ with the initial values $\left(p_{00}, p_{01}, p_{10}, p_{11}\right)=$ $(0.25,0.25,0.25,0.25)$ and $\lambda=100$. The desired convergence accuracy is $10^{-6}$. We repeat the simulation 1000 times. The results of means and standard errors are given in Tab. 2. It can be seen that estimates are very close to their true probabilities.

\section{Acknowledgements}

We would like to thank the two referees for their valuable comments and suggestions that improved the presentation of this paper. This research was supported by NSFC 10431010, NBRP 2005CB523301 and MSAR.

\section{References}

[1] C. Beeri, R. Fagin, D. Maier, M, Yannakakis, On the desirability of acyclic database schemes, J. Association for Computing Machinery 30 (1983) 479-513.

[2] P. J. Bickel, K. A. Doksum, Mathemetical Statistics. Holden-Day, Oakland, 1977.

[3] A. P. Dempster, N. M. Larid, D. B. Rubin, Maximum likelihood estimation from incomplete data via the EM algorithm(with disscussion), J. R. Stat. Soc. Ser. B. 39 (1977) 1-38.

[4] Z. Geng, K. Wan, F. Tao, Mixed graphical models with missing data and the partial imputation EM algorithm, Scan. J. of Stat. 27 (2000) 433-444.

[5] R. J. A. Little and D. B. Rubin, Statistical Analysis with Missing Data, 2nd Ed. Wiley, New York, 2002.

[6] S. Rassler, Statistical Matching. Lecture Notes in Statistics, 168, Springer, New York, 2002. 